\title{
An efficient meshless method based on the Moving Kriging interpolation for two-dimensional variable-order time-fractional mobile/immobile advection-diffusion model
}

\author{
Esmail Hesameddini ${ }^{1}$, Ali Habibirad ${ }^{1}$, Mohammad Hossein Heydari $^{1}$, and Reza Roohi ${ }^{2}$ \\ ${ }^{1}$ Shiraz University of Technology \\ ${ }^{2}$ Fasa University
}

April 28, 2020

\begin{abstract}
in this work, we introduce an efficient meshless technique for solving the two-dimensional variable-order time-fractional mobile/immobile advection-diffusion model with Dirichlet boundary conditions. The main advantage of this scheme is to obtain a global approximation for this problem which reduces such problems to a system of algebraic equations. To approximate the first and fractional variable-order against the time, we use the finite difference relations. The proposed method is based on the Moving Kriging (MK) interpolation shape functions. To discretization this model in space variables, we use the MK interpolation. Duo to the fact that the shape functions of MK have Kronecker's delta property, boundary conditions are imposed directly and easily. To illustrate the capability of the proposed technique on regular and irregular domains, several examples are presented in different kinds of domains and with uniform and nonuniform nodes. Also, we use this scheme to simulating anomalous contaminant diffusion in underground reservoirs.
\end{abstract}

\section{Hosted file}

Main.pdf available at https ://authorea.com/users/306619/articles/437594-an-efficient-meshlessmethod-based-on-the-moving-kriging-interpolation-for-two-dimensional-variable-order-timefractional-mobile-immobile-advection-diffusion-model 
figures/dom1/dom1-eps-converted-to.pdf 
figures/dom2/dom2-eps-converted-to.pdf 
figures/dom3/dom3-eps-converted-to.pdf 
figures/dom4/dom4-eps-converted-to.pdf 
figures/dom5/dom5-eps-converted-to.pdf 
figures/dom6/dom6-eps-converted-to.pdf 
figures/numexacex1/numexacex1-eps-converted-to.pdf

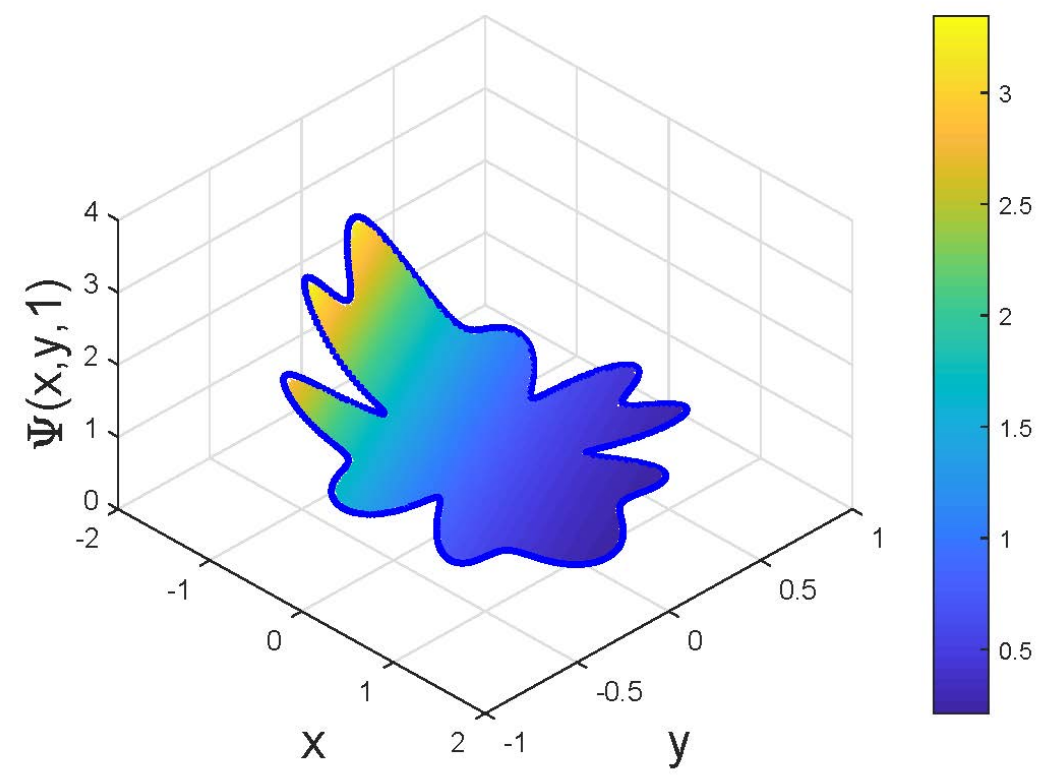



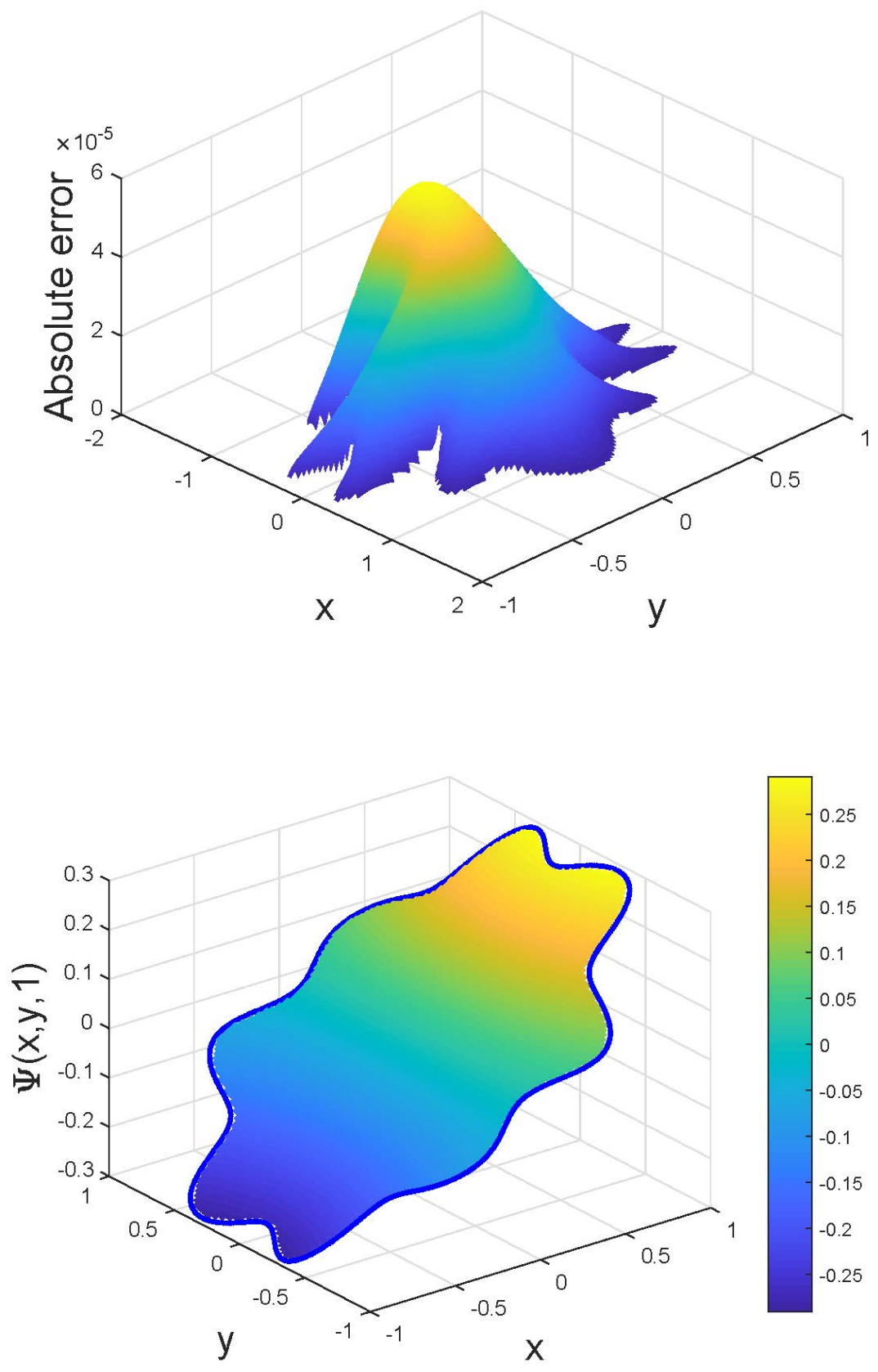

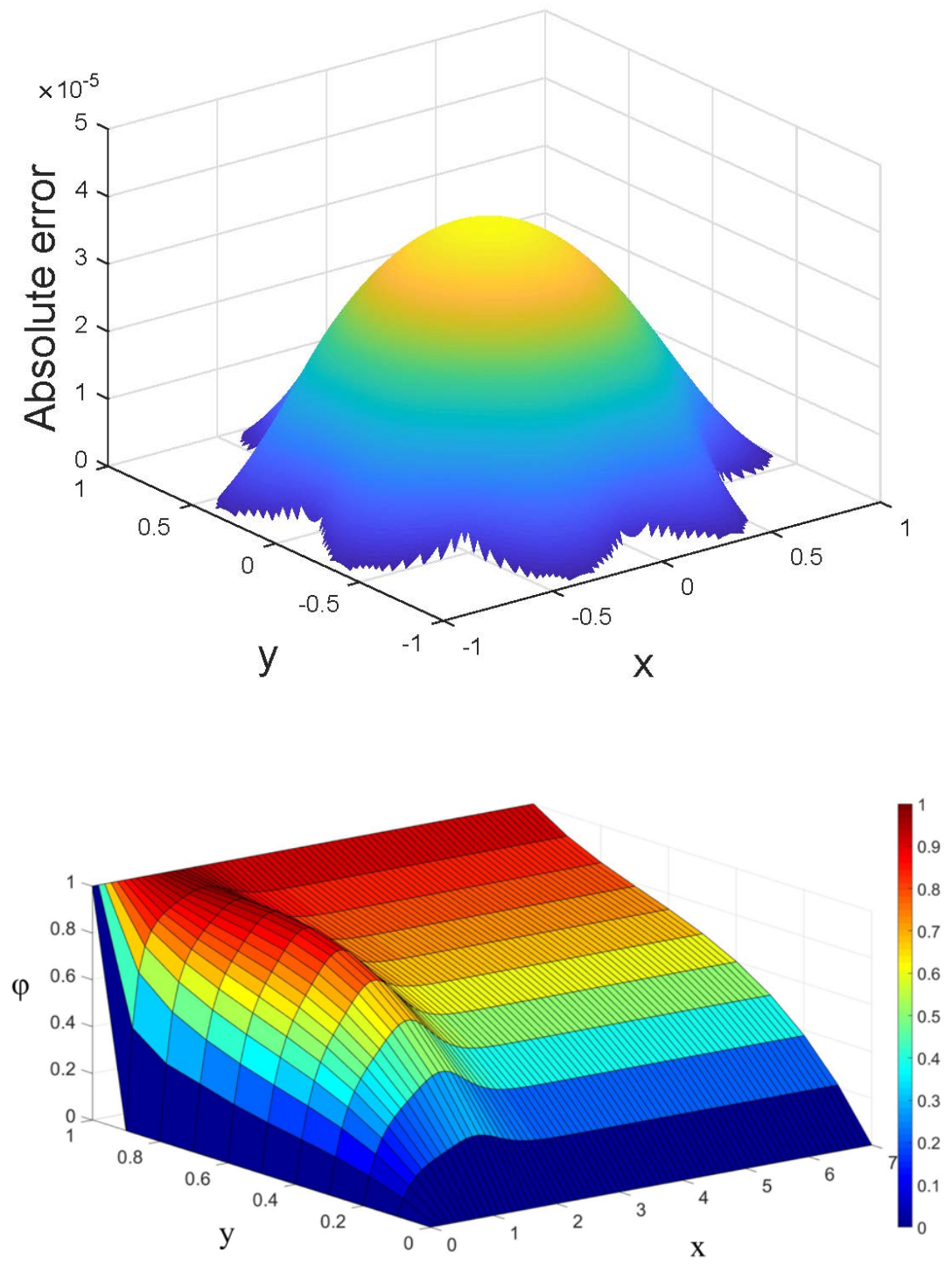


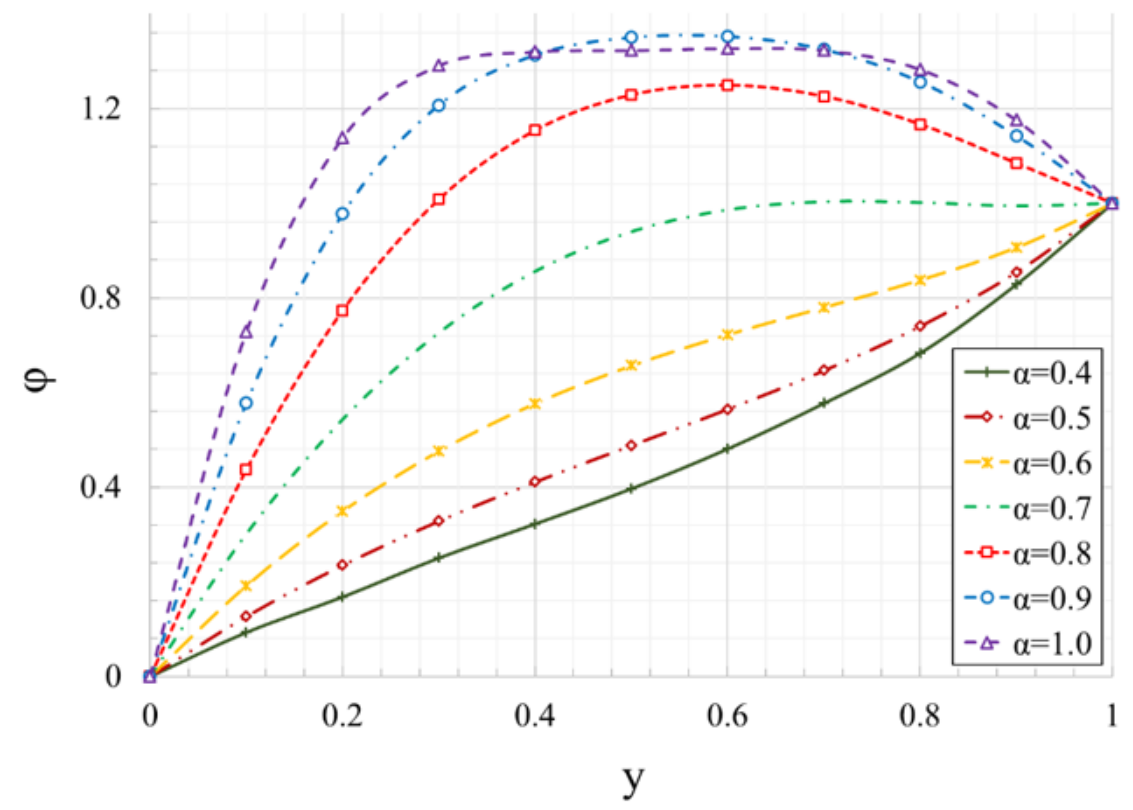

figures/errorex1/errorex1-eps-converted-to.pdf 